\title{
Prospective Study of 114 Consecutive Episodes of Staphylococcus aureus Bacteremia
}

\author{
Joseph M. Mylotte, Carolyn McDermott, \\ and Jody A. Spooner
}

\author{
From the Division of Infectious Diseases, Department of \\ Medicine and the Department of Microbiology, State \\ University of New York at Buffalo; and the Veterans \\ Administration Medical Center, Buffalo, New York
}

From 1 April 1983 to 31 October 1985, 114 episodes of Staphylococcus aureus bacteremia (SAB) were identified in 111 patients at the Buffalo Veterans Administration Medical Center. Only $14 \%$ of the episodes were community-acquired, and $29 \%$ were due to methicillin-resistant strains. The commonest foci of SAB were intravascular catheters $(33 \%)$, postoperative wounds $(11 \%)$, skin infections $(7 \%)$, and pulmonary infections $(7 \%)$. Complications were infrequent, with endocarditis in two patients and metastatic infection in one. Mortality due to SAB was $32 \%$, with no difference in mortality between communityacquired and hospital-acquired $\mathrm{SAB}$. Although not statistically significant, there was a trend of higher mortality for methicillin-resistant SAB $(42 \%)$ than for methicillin-sensitive SAB $(28 \%)$ and for patients with no focus of SAB $(43 \%)$ than for those with a defined primary focus $(28 \%)$ ). A review of studies of SAB published since 1940 revealed several trends. SAB is now predominately a nosocomial infection; intravascular-catheter infection has become the commonest cause of $\mathrm{SAB}$; with several exceptions, the risk of endocarditis in patients with $\mathrm{SAB}$ is low $(5 \%-20 \%)$; mortality due to SAB has decreased over the past 40 years but not over the past 10 years.

Staphylococcus aureus was recently characterized by Sheagren [1] as the "persistent pathogen." Despite the availability of potent antistaphylococcal antibiotics, infection due to $S$. aureus continues to cause considerable morbidity and mortality [2-4]. The most serious result of infection is $S$. aureus bacteremia (SAB).

Since 1940 many studies have been published dealing with $\mathrm{SAB}$ in general hospital populations [5-25]. Only three of these studies $[18,19,21]$ were performed prospectively. In one of these studies [19], which was performed by one of us (J. M. M.), 49 consecutive episodes of SAB were followed at two hospitals. Although this study provided useful information, the number of patients evaluated was relatively small and the findings may not have necessarily represented the overall picture of $\mathrm{SAB}$ at either hospital.

In the current study the results of a 30 -month

Received for publication 25 August 1986 and in revised form 20 January 1987.

This study was supported by the Veterans Administration.

Dr. Steven Gutman kindly provided access to laboratory records. Dr. John Sellick provided critical review of the manuscript. Sally Maier provided secretarial assistance.

Please address requests for reprints to Dr. Joseph M. Mylotte, Infectious Diseases (111F), Veterans Administration Medical Center, 3495 Bailey Avenue, Buffalo, New York 14215. prospective evaluation of all cases of SAB that occurred at the Veterans Administration Medical Center (VAMC) in Buffalo, New York, are reported. Our specific objectives included, but were not limited to: (I) determination of the risk of endocarditis in the subset of patients with community-acquired SAB in whom it has been retrospectively noted [15] that there is a high risk of endocarditis; (2) extension of a previous report [19] regarding the overall risk of endocarditis and metastatic infection in patients with $\mathrm{SAB}$; and (3) evaluation of the types of infections producing $\mathrm{SAB}$, with the intent of defining possible preventive measures. We also review studies of SAB published after 1940.

\section{Methods and Definitions}

All of the patients evaluated in this study were inpatients at the VAMC in Buffalo, New York. Two patients with a diagnosis of $\mathrm{SAB}$ who were transferred to the Buffalo VAMC from other institutions were not included in this study. The Buffalo VAMC is a 628-bed, tertiary-care, university-affiliated hospital with an additional 106 beds designated for long-term care. The Buffalo VAMC provides acute medical and surgical care; there are separate medical and surgical intensive care units. The VAMC also provides separate services for oncology, hemodialysis, rehabili- 
tation medicine, drug detoxification, psychiatry, neurology, and geriatrics.

Patients with SAB were identified by frequent monitoring of the blood-culture log book in the microbiology laboratory during the period from 1 April 1983 to 31 October 1985 . During the study period blood cultures were performed by injecting $5-10 \mathrm{~mL}$ of blood into a commercial two-bottle media system (BACTEC; Johnson Laboratories, Towson, Md. 21204). S. aureus was identified by use of standard microbiologic criteria. Antimicrobial susceptibility testing was performed by use of the disk diffusion method [26]. The detection of methicillin-resistant $S$. aureus (MRSA) strains was performed by susceptibility tests at $35^{\circ} \mathrm{C}$ for 48 hours [27]. Cultures of other body fluids and sites were performed in the usual manner. Intravenous catheter tips were cultured by use of the semiquantitative technique of Maki et al. [28].

$S A B$. Patients were considered to have $\mathrm{SAB}$ if two or more blood cultures were positive for $S$. aureus within a 24-hour period, or one blood culture was positive for $S$. aureus and the clinical course was consistent with staphylococcal infection. SAB was considered community-acquired if the first positive blood culture was obtained within 72 hours of admission or if there was clinical and cultural evidence of $S$. aureus infection at a body site at the time of admission. SAB was considered hospital-acquired if the first positive blood culture was obtained $\geqslant 72$ hours after admission and no clinical evidence of infection (fever, leukocytosis, signs, or symptoms) was present on admission.

Primary and secondary foci of $S A B$. A localized focus of staphylococcal infection was considered the source (primary focus) of SAB if signs and symptoms of infection or confirmatory physical findings antedated the bacteremia. Foci of staphylococcal infection in the CNS or joints were always considered to be secondary foci unless there was evidence of direct inoculation (surgery or trauma). A peripheral iv catheter was considered the focus of SAB if there was no other focus of infection identified and one or more of the following was present: inflammation at the site of insertion of the catheter; purulent drainage from the insertion site that on culturing, grew $S$. aureus; or a semiquantitative culture of the catheter [28] that was positive for $S$. aureus. A central venous catheter was considered to be the source of $\mathrm{SAB}$ if there was no other focus of infection and one or more of the following applied: the catheter had been in place for at least 72 hours, semiquantitative catheter culture was positive for $S$. aureus, or there was purulent drainage from the insertion site that, on culturing, grew $S$. aureus.

Endocarditis. Patients with $\mathrm{SAB}$ and one or more of the following were considered to have endocarditis: pathologic evidence of endocarditis at autopsy; a murmur in diastole, a new or changing murmur, or a characteristic regurgitant murmur; a murmur plus embolic phenomena; or evidence of endocarditis at operation confirmed by histologic examination.

Antibiotic treatment. The final decision as to the specific antibiotic prescribed and the duration of therapy was the responsibility of the primary care physician.

Outcome. Five categories of outcome were defined. (I) Death due to SAB. Patient deaths were attributed to staphylococcal infection if one or more of the following criteria pertained in the absence of another explanation for death: positive blood cultures for $S$. aureus at the time of death; a persistent focus of staphylococcal infection at death; persistent signs or symptoms of infection such as fever, leukocytosis, or hypotension; or death within the first 7 days of documentation of SAB without another explanation. (2) Death due to underlying disease during treatment of SAB. Patient deaths were attributed to underlying disease(s) during treatment of SAB if all of the following pertained: repeat blood cultures were sterile and there was evidence of control of infection manifested by resolution of or resolving fever, leukocytosis, and signs and symptoms at a specific body site and the underlying disease(s) was of a severity that death might be expected. (3) Recovery from SAB but ultimate death from underlying disease during the same admission. Patients were placed in this category if death occurred due to a defined underlying disease(s) after completion of therapy for SAB (with resolution of symptoms and signs of infection). (4) Recovered from SAB but remained hospitalized. Patients in this category successfully completed a course of therapy for SAB, but remained hospitalized due to other medical problems. (5) Recovery from SAB and discharged. Patients in this category successfully completed therapy for SAB and were discharged from the hospital.

By use of the aforementioned definitions, patients with $\mathrm{SAB}$ were observed and the following information was tabulated: age, hospital service, underlying diseases, primary focus of infection, development 
of complications (e.g., metastatic infection, endocarditis), antibiotic(s) administered, duration of therapy, and outcome. Each patient was followed until death occurred from SAB or underlying disease during the course of antibiotic therapy; in those patients who successfully completed therapy, follow-up was continued until discharge or for a maximum of 4 weeks after therapy in those who remained hospitalized. However, because complications of SAB may not be manifest for several weeks after discontinuation of therapy [29], the inpatient and outpatient records of all patients who completed therapy were reviewed after completion of the study to determine if there were any delayed complications.

Determination of the yearly frequency of SAB at the Buffalo VAMC and comparison with the yearly frequency of bacteremia due to other organisms was carried out by a review of the blood-culture log books from 1977 through 1985. By use of a microcomputer and data management software, the following information was tabulated for each patient with positive blood cultures: social security number, date of the cultures, ward location, number of blood-culture sets performed, number of blood-culture bottles that demonstrated bacterial growth, organism(s) isolated, and susceptibility-test results. If a patient had positive blood cultures for the same organism on several successive days, this was counted as only one bacteremic episode unless the period of time between positive cultures was $\geqslant 7$ days. For this analysis the presence of any Enterobacteriaceae, Pseudomonas species, and anaerobic bacteria in blood cultures was considered to represent a true bacteremia. Isolation of coagulase-negative staphylococci from a particular patient was considered to represent contamina- tion unless blood cultures performed on at least two separate days were positive and the organisms were detected within 48 hours of blood-culture inoculation [30]. Isolation of $S$. aureus in blood cultures was considered to document a true bacteremia in a particular patient if two or more sets of blood cultures drawn on the same day were positive or if single blood-culture sets drawn on several successive days were positive. The finding of $S$. aureus isolates that took $>48$ hours to grow or of only one positive bottle among one or more sets of cultures was considered to represent contamination. For the purpose of the present report, only data on the yearly rate of bacteremia for various bacterial species will be reported.

Comparisons of clinical characteristics were performed by $\chi^{2}$ analysis. Analysis of the rates of bacteremia over time was performed by linear regression. A $P$ value of $\leqslant .05$ was considered significant.

Studies of SAB in adults that were published from 1940 on were reviewed. The review included studies in which pediatric patients as well as adult patients were evaluated. However, studies dealing specifically with pediatric patients, cancer or hemodialysis patients, outbreaks of SAB, or only with bacteremia due to MRSA were not included. For each study reviewed, particular attention was paid to primary foci of SAB, complications, and mortality.

\section{Results}

The results of the review of the blood-culture log books are given in table 1 along with hospital demographic information for the period 1 January 1977 31 December 1985 . Increases in the yearly number Table 1. Annual rates of blood-culture processing and bacteremia at the Buffalo Veterans Administration Medical
Center, 1977-1985.

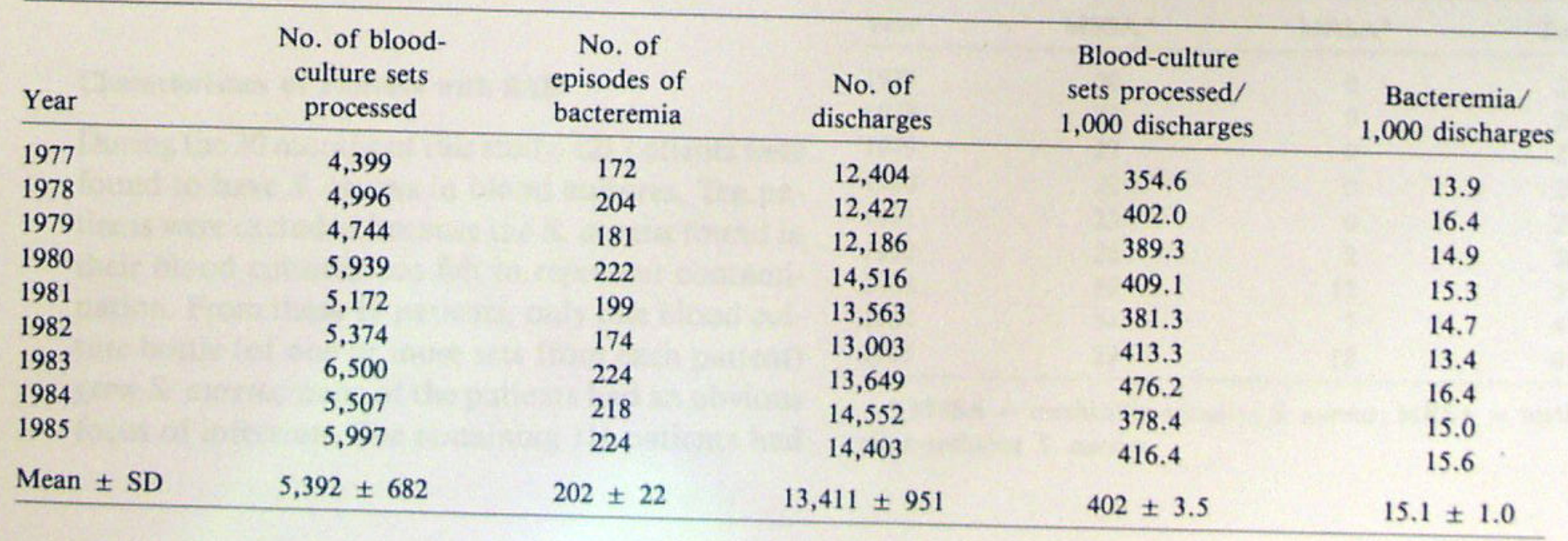


Table 2. Rate of bacteremia for the six most commonly isolated microorganisms at the Buffalo Veterans Administration Medical Center, 1977-1985.

\begin{tabular}{ccccccc}
\hline & \multicolumn{7}{c}{ No. of episodes of bacteremia with indicated organism/10,000 discharges } \\
\cline { 2 - 7 } Year & $\begin{array}{c}\text { Staphylococcus } \\
\text { aureus }\end{array}$ & $\begin{array}{c}\text { Escherichia } \\
\text { coli }\end{array}$ & $\begin{array}{c}\text { Streptococcus } \\
\text { pneumoniae }\end{array}$ & $\begin{array}{c}\text { Klebsiella } \\
\text { pneumoniae }\end{array}$ & $\begin{array}{c}\text { Proteus } \\
\text { mirabilis }\end{array}$ & $\begin{array}{c}\text { Pseudomonas } \\
\text { aeruginosa }\end{array}$ \\
\hline 1977 & 16.1 & 28.2 & 11.3 & 8.1 & 8.1 & 8.1 \\
1978 & 18.5 & 29.0 & 17.7 & 16.1 & 15.3 & 7.3 \\
1979 & 22.1 & 31.1 & 8.2 & 9.8 & 10.7 & 8.2 \\
1980 & 20.0 & 31.7 & 18.6 & 12.4 & 11.0 & 3.4 \\
1981 & 16.9 & 28.7 & 11.1 & 15.4 & 13.2 & 8.1 \\
1982 & 21.5 & 26.2 & 16.2 & 11.5 & 14.6 & 7.7 \\
1983 & 37.5 & 35.3 & 14.0 & 16.9 & 14.0 & 7.4 \\
1984 & 28.1 & 36.3 & 13.0 & 15.8 & 8.9 & 8.9 \\
1985 & 28.5 & 24.3 & 12.5 & 11.8 & 10.4 & 10.4 \\
\hline
\end{tabular}

NOTE. The correlation coefficient $(r)$ for each organism was: $S$. aureus, $+.730(P<.05) ;$ E. coli, $+.106 ; S$. pneumoniae, - .001; K. pneumoniae, +.401; P. mirabilis, $-.004 ;$ and $P$. aeruginosa, +.407.

of episodes of bacteremia detected and in hospital discharges paralleled the gradual increase in the yearly number of blood cultures processed during this period. However, the yearly rate of bacteremia showed little variation during the same period, with an average ( \pm SD) of $15.1 \pm 1.0$ episodes of bacteremia per 1,000 discharges.

The six bacterial species most commonly isolated from blood cultures between 1977 and 1985 are listed in table 2 along with the yearly rate of isolation of each organism per 10,000 hospital discharges. Although there was some variation in the yearly rate of bacteremia due to Escherichia coli, Streptococcus pneumoniae, Klebsiella pneumoniae, Proteus mirabilis, and Pseudomonas aeruginosa, simple linear regression revealed no significant increase or decrease in the individual rates of bacteremia during the 9year study period. However, there was a significant increase in the rate of $\mathrm{SAB}$ during the same period. Analysis of the yearly frequency of SAB is shown in table 3; the increase in frequency of $\mathrm{SAB}$ began in 1983 and was, in part, due to the appearance of episodes due to MRSA strains.

\section{Characteristics of Patients with SAB}

During the 30 months of this study, 121 patients were found to have $S$. aureus in blood cultures. Ten patients were excluded because the $S$. aureus found in their blood cultures was felt to represent contamination. From these 10 patients, only one blood culture bottle (of one or more sets from each patient) grew $S$. aureus; none of the patients had an obvious focus of infection. The remaining 111 patients had
114 episodes of SAB. Three patients had two separate episodes of SAB.

Sixteen $(14 \%)$ of the 114 episodes of SAB were community acquired. All but three patients were male, a finding consistent with the population studied. No primary focus was identified for six $(38 \%)$ of 16 episodes of community-acquired SAB and 17 $(17 \%)$ of 98 hospital-acquired episodes. Thirty $(26 \%)$ episodes of SAB were due to MRSA; all of these episodes were hospital acquired. The mean age ( \pm SD) of the 111 patients was $64.6 \pm 11.5$ years; only five patients were younger than 50 years. There was no significant difference in the mean age of patients with methicillin-susceptible SAB (MSSAB) and MRSA bacteremia (MRSAB) $-64.6 \pm 11.5$ and 65.5 \pm 11.0 years, respectively. All but two patients had at least one significant underlying disease. There was

Table 3. Annual frequency of Staphylococcus aureus bacteremia at the Buffalo Veterans Administration Medical Center, 1977-1985.

\begin{tabular}{cccc}
\hline & \multicolumn{3}{c}{ No. of episodes } \\
\cline { 2 - 4 } Year & MSSA & MRSA $^{*}$ & Total \\
\hline 1977 & 20 & 0 & 20 \\
1978 & 23 & 0 & 23 \\
1979 & 27 & 0 & 27 \\
1980 & 29 & 0 & 29 \\
1981 & 23 & 0 & 23 \\
1982 & 26 & 2 & 28 \\
1983 & 39 & 12 & 51 \\
1984 & 34 & 7 & 41 \\
1985 & 29 & 12 & 41 \\
\hline
\end{tabular}

* MSSA = methicillin-sensitive $S$. aureus; MRSA = methicillin-resistant $S$. aureus. 
Table 4. Foci of infection and selected characteristics of 114 episodes of Staphylococcus aureus bacteremia.

\begin{tabular}{|c|c|c|c|c|c|c|}
\hline \multirow[b]{3}{*}{ Focus } & \multirow{3}{*}{$\begin{array}{l}\text { Total no. } \\
\text { of episodes }\end{array}$} & \multicolumn{4}{|c|}{ No. of episodes with indicated characteristic } & \multirow{3}{*}{$\begin{array}{l}\text { Total no. } \\
\text { of deaths }\end{array}$} \\
\hline & & \multicolumn{2}{|c|}{ Acquisition } & \multirow{2}{*}{$\begin{array}{l}\text { Due to } \\
\text { MRSA }\end{array}$} & \multirow{2}{*}{$\begin{array}{c}\text { Death due } \\
\text { to } \mathrm{SAB}\end{array}$} & \\
\hline & & Community & Hospital & & & \\
\hline Intravascular catheter & 38 & 1 & 37 & 11 & 9 & 18 \\
\hline Wound & 13 & 0 & 13 & 4 & 4 & 4 \\
\hline Skin & 8 & 3 & 5 & 3 & 2 & 2 \\
\hline Lung & 8 & 1 & 7 & 2 & 6 & 6 \\
\hline Decubitus ulcer & 5 & 2 & 3 & 1 & 2 & 3 \\
\hline Genitourinary tract & 4 & 0 & 4 & 0 & 0 & 2 \\
\hline Pacemaker & 3 & 0 & 3 & 1 & 0 & 0 \\
\hline Arteriovenous shunt & 3 & 0 & 3 & 0 & 0 & 0 \\
\hline Bone & 2 & 1 & 1 & 1 & 0 & 1 \\
\hline Peritonitis & 2 & 1 & 1 & 1 & 1 & 1 \\
\hline Orthopedic pin & 1 & 0 & 1 & 1 & 0 & 0 \\
\hline Biliary tract & 1 & 1 & 0 & 0 & 0 & 0 \\
\hline Mediastinum & 1 & 0 & 1 & 0 & 1 & 1 \\
\hline Joint & 2 & 0 & 2 & 0 & 1 & 1 \\
\hline Unknown & 23 & 6 & 17 & 5 & 10 & 13 \\
\hline Total & 114 & 16 & 98 & 30 & 36 & 52 \\
\hline
\end{tabular}

NOTE. Abbreviations: $\mathrm{MRSA}=$ methicillin-resistant $S$. aureus; $\mathrm{SAB}=S$. aureus bacteremia.

an average of 1.9 diseases per patient, and the percentage of patients with each disease was as follows: alcoholism $(19 \%)$, malignant solid tumors $(17 \%)$, coronary artery disease $(14 \%)$, chronic renal failure $(13 \%)$, cirrhosis $(13 \%)$, diabetes $(12 \%)$, chronic lung disease $(11 \%)$, and hematologic cancers $(5 \%)$.

\section{Foci of SAB}

The primary foci of SAB are given in table 4 . The single commonest primary focus was an infected iv catheter. These cases are discussed in more detail below. SAB secondary to pneumonia was associated with the highest mortality rate $(75 \%)$. Patients in whom no focus was identified had an overall mortality rate of $42 \%$. Infections of the lung, skin, and soft tissue and those due to iv catheters, wounds, and unknown foci accounted for $80 \%$ (91 of 114) of all episodes of SAB; $80(88 \%)$ of these 91 episodes were hospital-acquired infections. In this group of 91 patients, the relation between the focus of SAB and underlying disease was analyzed. Five $(63 \%)$ of eight patients with skin or soft tissue infection were diabetic; three $(38 \%)$ of eight patients with pneumonia had cancer; $14(37 \%)$ of 38 patients with intravascular catheter infection had cancer; and eight $(35 \%)$ of 23 patients with an unidentified focus of SAB had cirrhosis. For all foci except wound infections, SAB occurred more commonly on the medi- cal service than the surgical service; overall, 63 episodes of SAB occurred on the medical service, whereas 18 occurred on the surgical service. The remaining 23 episodes occurred on several other services, with no one service predominating.

There were 38 episodes of SAB due to an infected intravascular catheter. Twenty-eight of these cases have been previously reported [31]. There were 20 peripheral-catheter infections, of which 18 were located in peripheral veins in the upper extremities, one in the radial artery, and one in the femoral vein. Eighteen subclavian vein catheters became infected; 16 were standard central venous catheters, and two were Hickman catheters. The criteria used to make a diagnosis of intravascular catheter-associated SAB are given in table 5 . Nine $(24 \%)$ of 38 patients died as a direct result of catheter-induced SAB. In these nine patients the duration of therapy before death ranged from 0 to 11 days. There was no clinical evidence of endocarditis in any of these nine patients. However, endocarditis was suspected in one patient with a prosthetic aortic valve and metastatic lung cancer in whom SAB developed secondary to an infected peripheral catheter. After 1 day of therapy, the patient died, but an autopsy was not performed. Because of the high risk of endocarditis in patients with $\mathrm{SAB}$ and a prosthetic valve, it was presumed that endocarditis was present. An autopsy was performed on only one of the other eight patients who 
died, and no evidence of endocarditis was noted. Five other patients with catheter-associated SAB died of their underlying disease during the same admission after recovering from the SAB, and three patients died of their underlying disease while receiving treatment for SAB. Autopsies were not performed on these latter eight patients; none had clinical evidence of endocarditis. Thus, the overall mortality rate in this group of patients was $45 \%$ (17 of 38 ). For the 26 patients who recovered, the mean duration of antibiotic therapy was 13.8 days (range, 5-28 days). Only five patients received treatment for $\geqslant 21$ days. No complications were noted in this group of 26 patients.

\section{Bacteremia with MRSA}

During the study period 30 episodes of MRSAB occurred in 28 patients. The foci of infection of these 30 episodes included an iv catheter in 11 , an unknown site in five, skin in three, a wound in four, the lung in two, and miscellaneous sites in five. The mortality rate due to MRSAB was $42 \%$. Mortality was higher on the surgical service (six deaths [55\%] in 11 episodes) than the medical service (four deaths [29\%] in 14 episodes; $P>.1$ by $\chi^{2}$ ). Although this difference was not statistically significant, it was large enough to warrant evaluation of the initial antibiotic therapy prescribed. This evaluation revealed that $10(71 \%)$ of 14 patients on the medical service received vancomycin as initial therapy, while six $(55 \%)$ of 11 patients on the surgical service received vancomycin initially; this difference was not significant $(P>10)$.

The relation of antibiotic therapy prescribed to outcome of MRSAB was also analyzed. Although the mortality rate in patients receiving vancomycin with or without a second agent was lower $(32 \%$; six of 19) than the mortality rate in patients who received therapy that did not include vancomycin $(50 \%$; three of six), this difference was not statistically significant. These findings suggested that outcome for patients with MRSAB was improved when vancomycin was part of the therapeutic regimen. Since only slightly more than $50 \%$ of the patients with MRSAB on the surgical service received vancomycin, this may explain, in part, the higher mortality in this group. The lack of significant improvement in the mortality rate among patients with MRSAB treated with vancomycin may be due to the small number of patients in the analysis.

A secondary focus of infection was identified in
Table 5. Criteria used to make a diagnosis of intravascular-catheter-associated Staphylococcus aureus bacteremia (SAB).

\begin{tabular}{lcc}
\hline & $\begin{array}{c}\text { No. of patients with } \\
\text { infection of indicated } \\
\text { type of catheter* }\end{array}$ \\
\cline { 2 - 3 } $\begin{array}{c}\text { Peripheral } \\
(n=20)\end{array}$ & $\begin{array}{c}\text { Central venous } \\
(n=18)\end{array}$ \\
\hline Criterion & 16 & 0 \\
\hline $\begin{array}{l}\text { Inflammation with no other } \\
\text { focus of SAB }\end{array}$ & 3 & 8 \\
$\begin{array}{l}\text { Purulent drainage from } \\
\text { insertion site }\end{array}$ & $1 / 1$ & $12 / 12$ \\
$\begin{array}{l}\text { Semiquantitative catheter } \\
\text { culture (no. positive/ } \\
\text { no. cultured) }\end{array}$ & 0 & 3 \\
\hline No other focus of SAB ${ }^{\dagger}$ & & \\
\hline
\end{tabular}

* Some patients had more than one criterion and as a result the numbers add up to more than the number of episodes.

$\dagger$ No inflammation or drainage; no catheter culture performed.

only one patient. He was a 66 -year-old man who underwent an open reduction and internal fixation of a fractured acetabulum. Ten days after surgery an infection developed at the insertion site of the pin being used for internal fixation. Drainage from around the pin as well as blood cultures grew MRSA. The pin was removed and the patient received 2 weeks of vancomycin therapy despite the recommendation by an infectious diseases consultant for 4 weeks of parenteral therapy. Nine weeks after therapy was discontinued, the patient developed fever and was readmitted. Gallium scan and computed tomography of the vertebral skeleton revealed changes consistent with osteomyelitis at the L4-L5 level. Blood cultures again yielded MRSA. He was treated with vancomycin and rifampin for 3 weeks and was discharged to a nursing home. Our belief that the vertebral bone was seeded during the first episode of $\mathrm{SAB}$ and represented a secondary focus is based on the absence of an intercurrent illness that would have been associated with bacteremia and recurrence of MRSA in blood cultures.

\section{Endocarditis in Patients with SAB}

The diagnosis of endocarditis was made in only two of the 111 patients with SAB. One of the patients with a prosthetic heart valve and SAB was described in a previous section. The other patient was a 71year-old man with a history of stroke who was admitted with fever and confusion. Blood cultures per- 
formed at the time of admission grew $S$. aureus, but no focus of infection was identified. The patient was treated iv with nafcillin. A lumbar puncture was performed on the fifth day of hospitalization, and cultures of the CSF yielded a few colonies of $S$. aureus. The dose of nafcillin was increased from $6 \mathrm{~g}$ to 12 $\mathrm{g}$ on the sixth day of hospitalization. The patient died on the seventh day of hospitalization with intractable hypotension, acidosis, and persistent fever and leukocytosis. Autopsy revealed acute endocarditis of the mitral valve with multiple infarcts in the kidneys and spleen.

\section{Outcome of SAB}

Thirty-six (32\%) of 111 patients died due to SAB; four of the 36 patients received no treatment, and three of the four died within 24 hours after blood cultures were performed. For the remaining 32 patients, SAB was determined to be the cause of death on the basis of the following criteria: persistently positive blood cultures (two patients), persistent focus of staphylococcal infection (11 patients), persistent signs and symptoms of infection (15 patients), and death within 7 days of the first positive blood culture for which there was no other explanation (four patients). Only two autopsies were performed: one patient was found to have acute endocarditis of the mitral valve as previously detailed, and the other had no evidence of endocarditis or metastatic infection. In addition to the 36 patients who died as a direct result of SAB, 17 others died either of an underlying disease after successful treatment of SAB ( $n=10)$ or of underlying disease during treatment $(n=7)$. Autopsies were performed on three of these 17 patients, and none had evidence of endocarditis.

Fifty-eight patients with 59 episodes of SAB recovered; 24 of these patients remained hospitalized for 4 weeks after which follow-up was stopped, and 34 of the patients were discharged. Of the 34 patients who were discharged, all but four left the hospital within 7 days of discontinuation of antibiotic treatment for SAB. In order to determine if there were late complications of SAB, especially in those discharged, follow-up was sought in these 58 patients by retrospective review of hospital and clinic records after completion of the study in October 1985. This follow-up was performed in January 1986. No charts were available for 14 patients, but review of data from the medical administrative service of the VAMC revealed that three of the 14 were subsequently dis- charged, seven died during the same hospital admission in which SAB occurred, and there was no information for the remaining four patients. Another four patients were still in the hospital at the time of the follow-up, and they had no evidence of complications. The records of the remaining 40 patients were available for review. The mean duration of time from the onset of SAB to the latest notation in the record for these 40 patients was 34.8 weeks (range, 3-112 weeks). Ten of the 40 were found to have died, but there was no evidence of recurrent SAB or $S$. aureus infection. In the remaining 30 patients, there was no evidence of recurrent SAB, metastatic infection, or other complications.

Mortality directly due to MRSAB (42\%) was not significantly different from that due to MSSAB $(28 \%)$. Mortality secondary to community-acquired SAB $(31 \%)$ was similar to that due to hospitalacquired SAB (32\%). The mortality due to SAB in patients without an identifiable focus $(43 \%)$ was higher than in those with a primary focus $(28 \%)$, but this difference was not significant. The mortality rate directly due to $\mathrm{SAB}$ in patients younger than 60 years was $25 \%$ and that in those 60 years or older was $35 \%(P>.10)$. The rates of mortality due to SAB in patients with various underlying diseases were as follows: alcoholism (29\%), malignant solid tumors $(41 \%)$, coronary artery disease $(38 \%)$, chronic renal failure $(43 \%)$, cirrhosis $(35 \%)$, diabetes $(31 \%)$, chronic lung disease $(50 \%)$, and hematologic malignancies $(0 \%)$. There was a trend of higher mortality due to $\mathrm{SAB}$ in patients with three or more underlying diseases than in those with one or two underlying diseases ( $41 \%$ vs. $33 \%$ ), but this difference was not statistically significant.

The relation between the duration of antimicrobial therapy and outcome is given in table 6 . Almost all patients were treated with a single agent. It is apparent from the data that once a patient received therapy for at least 14 days, the chance of succumbing to infection was minimal; only two $(4 \%)$ of 51 patients who were treated for $\geqslant 14$ days died of infection. On the other hand, $34(94 \%)$ of the 36 patients who died of SAB had received $<14$ days of treatment at the time of death. Twenty-three $(67 \%)$ of these 34 received $\leqslant 7$ days of antibiotic therapy at the time of death; four patients died before treatment could be initiated. With the exclusion of the latter four patients, all of the patients who died of SAB were receiving antibiotic therapy at the time of death.

A comparison of several clinical characteristics of 
Table 6. Relation between duration of treatment and outcome of Staphylococcus aureus bacteremia in 106 patients.

\begin{tabular}{lcc}
\hline & $\begin{array}{c}\text { No. of patients (mean } \pm \mathrm{SD}) \\
\text { receiving treatment for } \\
\text { indicated period }\end{array}$ \\
\cline { 2 - 3 } Outcome & $<14 \mathrm{~d}$ & $\geqslant 14 \mathrm{~d}$ \\
\hline Died of infection & $\begin{array}{l}4.9 \pm 3.5 \\
(n=34)\end{array}$ & 26 \\
Recovered & $9.1 \pm 3.1$ & $19.6 \pm 5.5$ \\
& $(n=21)$ & $(n=49)$ \\
\hline
\end{tabular}

NOTE. Five patients who died of their underlying diseases during treatment were excluded.

the 49 patients who recovered after $\geqslant 14$ days of therapy and the 34 patients who died after $<14$ days of therapy was performed. In patients who died during the first 2 weeks of therapy, there were significantly more infections due to MRSA ( $38 \%$ vs. $18 \%$; $P<.05$ by $\left.\chi^{2}\right)$ and infections in which no focus was identified ( $26 \%$ vs. $10 \% ; P=.05)$. There was no significant difference between the two groups in regard to mean age, mean number of underlying diseases, frequency of specific foci of infection, frequency of community-acquired $\mathrm{SAB}$, or hospital service. In the group of patients who received $<14$ days of antibiotic treatment, a comparison of the clinical characteristics of those who died of SAB and those who recovered was performed. The only significantly different characteristic was the frequency of catheter-associated SAB: $62 \%$ of all infections in those who recovered were catheter-associated vs. $32 \%$ in those who died $(P<.05)$.

\section{Literature Review}

\section{Studies Published Between 1940 and 1960}

Table 7 lists selected findings of studies of SAB published between 1940 and 1960. In 1941 Skinner and Keefer [5] published the Boston City Hospital experience with SAB over 7 years. This study was representative of SAB in the preantibiotic era. The mortality rate was $82 \%$; however, most patients in this study received no antimicrobial therapy.

In 1957 Wilson and Hamburger [6] reported an analysis of 55 cases of staphylococcal bacteremia at the Cincinnati General Hospital (five patients had bacteremia due to coagulase-negative staphylococci) that occurred between 1940 and 1954 . The $64 \%$ rate of endocarditis in this study is the highest rate reported secondary to SAB. Twenty-four $(69 \%)$ of the 35 patients with endocarditis had underlying valvular heart disease. As a result of the high rate of endocarditis reported in this study, it became dogma that all patients with $\mathrm{SAB}$ should receive prolonged (6-week) antimicrobial therapy [32]. However, such a general approach based on these data may not have been entirely appropriate, given the high rate of underlying valvular heart disease in the study population. A more appropriate conclusion might have been that the risk of endocarditis in patients with valvu-

Table 7. Selected aspects of studies of Staphylococcus aureus bacteremia (SAB) published between 1941 and 1960.

\begin{tabular}{|c|c|c|c|c|c|c|c|}
\hline $\begin{array}{l}\text { Years of } \\
\text { study } \\
\text { [reference] }\end{array}$ & $\begin{array}{l}\text { No. } \\
\text { of } \\
\text { cases }\end{array}$ & $\begin{array}{l}\text { Community- } \\
\text { acquired } \\
\text { cases }(\%)\end{array}$ & Primary focus, $\%$ & $\begin{array}{l}\text { Patients with } \\
\text { endocarditis } \\
(\%)\end{array}$ & $\begin{array}{l}\text { Patients with } \\
\text { complications } \\
(\%)\end{array}$ & $\begin{array}{l}\text { Mortality } \\
\text { rate } \\
(\%)\end{array}$ & $\begin{array}{l}\text { Deaths } \\
\text { due to } \\
\text { SAB }(\%)\end{array}$ \\
\hline $1933-1940$ [5] & 122 & $?$ & $\begin{array}{l}\text { Skin, } 47 \text {; lung, } 25 ; \text { bone, } 9 ; \\
\text { genitourinary tract, } 9 ; \\
\text { unknown, } 10\end{array}$ & 5.7 & 82 & 82 & NS \\
\hline $1940-1954$ [6] & 55 & 100 & NS & 64 & 38 & 71 & NS \\
\hline $1940-1956[8]$ & 109 & NS & NS & * & 11 & 34 & NS \\
\hline $1936-1955[10]$ & 338 & NS & NS & 7 & NS & 78 & 72 \\
\hline $1951-1958$ [9] & 100 & 50 & $\begin{array}{l}\text { Skin, } 21 \text {; lung, } 21 \text {; post- } \\
\text { operative, } 22 \text {; genito- } \\
\text { urinary, } 17\end{array}$ & 8 & NS & 64 & NS \\
\hline $1957-1958$ [7] & 201 & 48 & Skin, 32 ; lung, 5 & NS & NS & 41 & NS \\
\hline $1950-1957$ [11] & 86 & 53 & $\begin{array}{l}\text { Skin, 23; intravascular } \\
\text { catheter, 23; lung, 17; } \\
\text { unknown, } 16\end{array}$ & 15 & NS & 58 & NS \\
\hline
\end{tabular}

NOTE. NS $=$ not stated.

* Cases of endocarditis excluded. 
Table 8. Selected findings of studies of Staphylococcus aureus bacteremia (SAB) published between 1961 and 1976.

\begin{tabular}{lccccccc}
$\begin{array}{l}\text { Years of } \\
\text { study } \\
\text { [reference] }\end{array}$ & $\begin{array}{c}\text { No. } \\
\text { of } \\
\text { cases }\end{array}$ & $\begin{array}{c}\text { Community- } \\
\text { acquired } \\
\text { cases (\%) }\end{array}$ & Primary focus, \% & $\begin{array}{c}\text { Patients with } \\
\text { endocarditis } \\
(\%)\end{array}$ & $\begin{array}{c}\text { Patients with } \\
\text { complications } \\
(\%)\end{array}$ & $\begin{array}{c}\text { Mortality } \\
\text { rate } \\
(\%)\end{array}$ & $\begin{array}{c}\text { Deaths } \\
\text { due to } \\
\text { SAB }(\%)\end{array}$ \\
\hline $1952-1965[12]$ & $185^{*}$ & 52 & $\begin{array}{c}\text { Skin, 35; wound, 18; } \\
\text { lung, 9; intravascular } \\
\text { catheter, 6 }\end{array}$ & 6 & 35 & 42 & NS \\
$1957-1966[13]$ & 2,027 & 38 & $\begin{array}{c}\text { Skin, 20; lung, 5; } \\
\text { unknown, 40 }\end{array}$ & 3 & NS & 43 & NS \\
$1969-1971[14]$ & 42 & 31 & NS & 14 & NS & 47 & NS \\
$1969-1973[15]$ & 105 & 54 & NS & 25 & 44 & 21 & 36 \\
\hline
\end{tabular}

NOTE. NS $=$ not stated.

* Twenty-five cases were due to coagulase-negative staphylococci.

lar heart disease and SAB is high and that such patients should be treated as if they have endocarditis. In patient populations without a high rate of valvular heart disease, such as that described by Skinner and Keefer [5], the rate of endocarditis was considerably lower.

Faber et al. [7] published a report of 201 episodes of SAB collected from 86 Danish hospitals in 1957 and 1958. Forty-one percent of the patients died. This study documented a higher mortality rate for hospital-acquired SAB $(55 \%)$ than for community-acquired SAB $(28 \%)$. Shirger et al. [8] reviewed the Mayo Clinic experience with 109 episodes of SAB without endocarditis from 1940 to 1956 . The mortality rate in this study $(34 \%)$ was lower than that reported by Skinner and Keefer [5] and Wilson and Hamburger [6], probably because patients with endocarditis were excluded, metastatic complications were uncommon, and all patients received some type of antimicrobial therapy.

Waisbren and Abboud [9] reviewed 100 cases of SAB that occurred at the Milwaukee County General Hospital between 1951 and 1958. Although primary foci were similar to those reported in previous studies and the rate of endocarditis was low $(8 \%)$, the mortality rate was high $(64 \%)$. It was not clear from the data presented why the mortality rate was so high, given that effective antimicrobial therapy was performed and few patients had endocarditis.

Smith and Vickers [10] reported 338 cases of SAB at the University Hospitals in Iowa City between 1936 and 1955. The 19-year period of this study is the longest of any published study since 1940 . In 87 patients who received no antimicrobial treatment, the mortality rate was $90 \%$, whereas in the 251 who received antimicrobial therapy, the mortality rate was $55 \%$. An interesting finding made by these authors was that the introduction of a new effective agent led to an initial decrease in mortality, but with continued use resistance occurred and efficacy was lost.

Hassall and Rountree [11] reviewed 86 episodes of SAB that occurred between 1950 and 1957 at a large teaching hospital in Australia. In the patients with hospital-acquired SAB, the commonest primary focus was an iv infusion ( 20 of 40 episodes). This appears to be one of the earliest observations of the risk of SAB secondary to infected iv catheters. Unfortunately, the criteria used to identify a catheter as the source of SAB were not stated.

Several conclusions can be made from the studies published during the period 1940-1960. First, with or without antimicrobial therapy, the mortality rate usually exceeded $50 \%$, except in one study [8] in which cases of endocarditis were excluded. Second, $\geqslant 50 \%$ of episodes of SAB were due to communityacquired infection. Third, other than the excessively high rate of endocarditis reported by Wilson and Hamburger [6], the rate of endocarditis was remarkably similar among the studies. Fourth, there was close agreement in all studies in regard to common primary foci of SAB. However, the finding of a high rate of iv-catheter infection by Hassall and Rountree [11] was a noteworthy exception and was a harbinger of the high rate of iv-catheter-associated SAB observed in the 1970s and early 1980s.

\section{Studies Published Between 1961 and 1976}

Cluff and colleagues [12] reported 185 episodes of SAB seen at the Johns Hopkins Hospital from 1952 to 1965 (table 8 ). This was the second study to emphasize intravascular catheters as a major cause of $\mathrm{SAB}$. Despite the higher frequency of underlying diseases in this study than in the previous studies, the 
mortality was considerably lower. This was probably related, in part, to the availability of semisynthetic penicillin resistant to $\beta$-lactamase as well as to continuing improvements in patient care.

Jessen et al. [13] reported on 1,935 patients with SAB (essentially all Danish cases of staphylococcal bacteremia) that occurred during 1957-1966 (table 8 ). There was a high rate of hospital-acquired $\mathrm{SAB}$ $(62 \%)$. The overall mortality rate of $43 \%$ and the $4 \%$ rate of endocarditis were remarkably similar to those reported by Cluff et al. [12] in the United States during almost the same period.

Austin and Wallace [14] retrospectively reviewed 42 cases of SAB at the University of Washington Hospital in Seattle from 1 January 1969 to 1 September 1971 (table 8). They studied children as well as adults. In comparison to several studies [12, 15] of $\mathrm{SAB}$ in the same period, the frequency of community-acquired $\mathrm{SAB}$ ( $31 \%$ of all cases) was lower. All cases of endocarditis occurred in association with community-acquired SAB. It is important to note that these authors provided information suggesting that the duration of $\mathrm{SAB}$ in communityacquired infection was considerably longer than that of hospital-acquired SAB (median of 5 days vs. 1 day). They concluded that this difference in duration of SAB was a major factor in the high rate of endocarditis among the patients with communityacquired SAB. These authors also pointed out that in the absence of endocarditis, duration of therapy need not exceed 3 weeks. This statement was counter to the recommendation that all patients with $\mathrm{SAB}$ required 4-6 weeks of antimicrobial therapy because of the high risk of endocarditis $[6,29]$.

A frequently quoted study is that of Nolan and Beaty [15] (table 8). These authors reviewed 105 cases of SAB at the Harborview Medical Center in Seattle, Washington, between 1969 and 1973. It is noteworthy that $53 \%$ of community-acquired cases occurred in iv-drug abusers. In contrast to the low rate of endocarditis reported in previous studies (except the study by Wilson and Hamburger [6]), Nolan and Beaty reported a rate of $25 \%$, but the overall mortality rate was only $21 \%$, a considerable decrease from the $43 \%$ reported by Cluff et al. [12]. A major point made by Nolan and Beaty [15] was that most of the cases of endocarditis occurred in patients with community-acquired SAB in which no primary focus could be identified. From these findings the authors concluded that community-acquired $\mathrm{SAB}$ occurring in the absence of a primary focus was associated with a high risk of endocarditis. However, this conclusion was influenced by the fact that twothirds of their cases of community-acquired SAB associated with endocarditis occurred in drug abusers. This particular patient population is well known to have $\mathrm{SAB}$ without a primary focus $[33,34]$. Thus, the findings of Nolan and Beaty may not necessarily apply to a population without drug abusers.

Nolan and Beaty also suggested that the lower mortality rate reported was due to the availability of effective antibiotics and to other factors. However, SAB in drug addicts with or without endocarditis has a good prognosis [33-35]. This factor, coupled with the large number of drug addicts in this study, would tend to make the prognosis better than that observed in other investigations in which few or no drug abusers were studied. On the other hand, Nolan and Beaty suggested that patients with a defined focus of SAB and no secondary foci may not require prolonged (4- to 6-week) antibiotic therapy since the risk of endocarditis appeared to be low in this group. This was similar to the recommendation made by Austin and Wallace [14].

The findings in this second era of publications concerned with SAB can be summarized as follows. An improvement in overall survival was noted, but mortality in older patients (i.e., $>50$ years old) remained high $(>40 \%)$. The risk of endocarditis related to $\mathrm{SAB}$ remained in the range of $5 \%-10 \%$ (excluding the study of Nolan and Beaty [15]). There was again recognition and further emphasis that the iv catheter was an important focus of SAB [12]. This observation set the stage for a continuing debate of the risk of endocarditis in patients with catheterinduced SAB [31, 36, 37]. Nolan and Beaty [15] also emphasized that $\mathrm{SAB}$ in the subset of patients with a defined focus without metastatic infection can be treated with 2-3 weeks of antibiotic therapy rather than 4-6 weeks. Finally, Nolan and Beaty [15] put forth the concept that community-acquired $\mathrm{SAB}$ without a focus of infection was associated with a significant risk of endocarditis. As previously mentioned, this conclusion may have been biased by the large number of drug addicts in their study.

\section{Studies Published Since 1976}

Results of studies published since 1976 are summarized in table 9. Shah and Watanakunakorn [16] updated the experience with $\mathrm{SAB}$ at the Cincinnati General Hospital and contrasted it with the earlier 
Table 9. Selected findings in studies of Staphylococcus aureus bacteremia (SAB) published since 1976.

\begin{tabular}{|c|c|c|c|c|c|c|c|}
\hline $\begin{array}{l}\text { Years of } \\
\text { study } \\
\text { [reference] }\end{array}$ & $\begin{array}{l}\text { No. } \\
\text { of } \\
\text { cases }\end{array}$ & $\begin{array}{l}\text { Community- } \\
\text { acquired } \\
\text { cases }(\%)\end{array}$ & Primary focus, $\%$ & $\begin{array}{l}\text { Patients with } \\
\text { endocarditis } \\
(\%)\end{array}$ & $\begin{array}{l}\text { Patients with } \\
\text { complications } \\
(\%)\end{array}$ & $\begin{array}{l}\text { Mortality } \\
\text { rate } \\
(\%)\end{array}$ & $\begin{array}{l}\text { Deaths } \\
\text { due to } \\
\text { SAB (\%) }\end{array}$ \\
\hline $1975-1977[16]$ & 134 & 40 & $\begin{array}{l}\text { Skin, } 31 \text {; intravascular } \\
\text { catheter, 18; lung, } 13 \text {; } \\
\text { bone, } 6 \text {; unknown, } 26\end{array}$ & 16 & NS & 30 & 20 \\
\hline $1977-1980[17]$ & 61 & 46 & $\begin{array}{l}\text { Intravascular catheter, } 21 \text {; } \\
\text { ulcer, } 13 \text {; wound, 13; } \\
\text { bone, } 5 \text {; skin, } 5\end{array}$ & 20 & NS & 36 & NS \\
\hline $1976-1979[18]$ & 76 & 54 & $\begin{array}{l}\text { Skin, 32; intravascular } \\
\text { catheter, 22; bone, } 11 \text {; } \\
\text { lung, } 8 \text {; unknown, } 22\end{array}$ & 33 & 53 & 41 & NS \\
\hline $1978-1980$ [19] & 49 & 31 & $\begin{array}{l}\text { Skin, } 10 \text {; intravascular } \\
\text { catheter, } 29 \text {; bone, } 10 \text {; } \\
\text { unknown, } 22\end{array}$ & 4.3 & NS & 24 & 15 \\
\hline $1976-1980[20]$ & 169 & 41 & $\begin{array}{l}\text { Wounds }+ \text { intravascular } \\
\text { catheter, } 50\end{array}$ & 11 & 11 & NS & 17 \\
\hline $1969-1983[21]$ & 400 & 30 & $\begin{array}{l}\text { Intravascular catheter, } 23 \text {; } \\
\text { wound, } 20 \text {; shunt, } 14 \text {; } \\
\text { skin, } 14\end{array}$ & 7 & NS & 24 & 13 \\
\hline $1980-1981$ [22] & 39 & 13 & $\begin{array}{r}\text { Intravascular catheter, } 51 ; \\
\text { unknown, } 10 ; \text { shunt, } 15\end{array}$ & 0 & 36 & 30 & 11 \\
\hline $1979-1982[23]$ & 103 & 38 & $\begin{array}{l}\text { Intravascular catheter, } 25 \\
\text { skin, } 41 \text {; lung, } 3\end{array}$ & 14 & 23 & 43 & NS \\
\hline $1977-1981[24]$ & 390 & NS & $\begin{array}{l}\text { Intravascular catheter, 10; } \\
\text { lung, 19; skin, 7; } \\
\text { wound, } 9 \text {; unknown, } 32\end{array}$ & 5 & NS & 31 & 16 \\
\hline $1965-1982[25]$ & 248 & 100 & NS & 31 & NS & NS & 19 \\
\hline
\end{tabular}

NOTE. NS $=$ not stated.

report from the same hospital by Wilson and Hamburger [6]. Twenty patients $(16 \%)$ developed endocarditis; 13 of the 20 patients with endocarditis acquired $\mathrm{SAB}$ in the community, and eight of these 13 were iv-drug abusers. Of note is that four $(18 \%)$ of 22 patients with iv-catheter-associated SAB developed endocarditis; this is the highest rate reported for this particular subgroup. Mortality was significantly greater for patients over age 50 years $(51 \%)$ than for those 50 years or younger $(13 \%)$. The major differences between this report and that of Wilson and Hamburger [6] were that the mortality rate was considerably lower ( $71 \%$ vs. $30 \%)$ and the rate of endocarditis decreased fourfold ( $65 \%$ vs. $16 \%)$. The main reason for the decrease in the rate of endocarditis, in our opinion, was the smaller number of patients with rheumatic valvular disease in the study by Shah and Watanakunakorn [16] than in that of Wilson and Hamburger (38\% vs. $2 \%$ ) [6].

Cooper and Platt [17] reported the experience with SAB at the New England Deaconess Hospital (Boston) between August 1977 and August 1980. Since this hospital is the principal admitting institution for the Joslin Diabetes Foundation, the authors compared SAB in diabetics with that in nondiabetics. Of the 61 patients with SAB, $27(44 \%)$ were diabetic. As a result of the predominance of diabetics in the study population, infections of foot ulcers and osteomyelitis were frequent primary foci of SAB. However, infected iv catheters were the single commonest focus. Endocarditis occurred in $20 \%$ of all episodes of SAB; 11 of the 12 episodes of endocarditis were community acquired. Six episodes of endocarditis occurred in the absence of an identifiable primary focus; the remaining six episodes occurred in patients with a primary focus, and all six were diabetics. Only one episode of endocarditis occurred among $13 \mathrm{pa}$ tients with iv-catheter-induced SAB. Comparing diabetics and nondiabetics, the authors found no difference in mortality, but diabetics with $\mathrm{SAB}$ were more likely to have endocarditis in the presence of a primary focus.

Several important points were made by Cooper and Platt in the discussion of their findings. They noted that the $36 \%$ overall mortality was higher than that in other studies $(21 \%$ in the study of Nolan and 
Beaty [15]). These authors, however, also pointed out that $20 \%$ of their patients with SAB had metastatic cancer and a considerable proportion had other debilitating diseases. Thus, there would be a tendency toward a higher overall mortality rate in this study than in studies of patients with a favorable prognosis, e.g., drug addicts [35]. They also noted that endocarditis occurred in patients both with and without a primary focus. However, one must be cautious in the interpretation of this finding. All cases of endocarditis related to a primary focus occurred in the diabetic group. Five of the six cases of endocarditis in diabetics were related to chronic infection in a distal extremity. Cooper and Platt [17] were careful to point this out. Thus, this study suggested that diabetics with chronic, indolent infections of distal extremities may be at increased risk for complications such as endocarditis and probably should receive a prolonged (at least 4- to 6-week) course of antibiotic therapy. The results of the study supported the concepts that patients with community-acquired $\mathrm{SAB}$ without a focus are at risk for endocarditis and that those with iv-catheter-induced SAB have a minimal risk of endocarditis.

Mirimanoff and Glauser [18] reviewed 76 cases of SAB at the University Medical Center in Lausanne, Switzerland, between January 1976 and July 1979. It is important to note that $53 \%$ of the episodes of SAB were associated with metastatic complications and $33 \%$ of the patients developed endocarditis. The rate of endocarditis in patients without a primary focus $(12$ of $17 ; 71 \%$ ) was significantly greater than the rate in those with an identifiable focus ( 13 of 59 ; $22 \%$ ). However, one-half of the cases of endocarditis were associated with a primary focus. Only one $(6 \%)$ of 16 patients with intravascular catheter-induced SAB developed endocarditis. As in other studies mortality was significantly lower in patients 50 years old or younger $(7 \%)$ than in those older than 50 years $(47 \%)$. Unlike previous studies in which high rates of endocarditis were found in populations with significant risk factors for developing endocarditis, e.g., valvular heart disease [6] or drug addiction [15], the population studied by Mirimanoff and Glauser [18] did not have these predisposing factors present. The explanation for the high rate of endocarditis in their study is unclear. Nevertheless, this study again verified the considerable risk of endocarditis in patients without a primary focus of SAB but also suggested that patients with a defined focus were at risk.

Mylotte et al. [19] prospectively evaluated 47 pa- tients with 49 consecutive episodes of SAB at two institutions between September 1978 and June 1980 . Complications were uncommon: only two patients $(4.3 \%)$ developed endocarditis, and metastatic infection was also infrequent. Of importance was that $29 \%$ of the episodes of SAB were related to infected iv catheters. In this subgroup of patients, endocarditis was not recognized and no patients died of SAB. Mortality rates were lower than those in other studies with similar patient populations [16, 22, 24].

Hedstrom and Christensson [20] retrospectively reviewed 169 cases of $\mathrm{SAB}$ that occurred at the University Hospital in Lund, Sweden, from 1976 to 1980. The findings, in terms of the types of primary foci of SAB and rates of endocarditis and mortality, were similar to those in other studies recently reported. Gransden and colleagues [21] prospectively evaluated 400 patients with SAB at a hospital in London, England. The findings in regard to the primary foci of SAB and rates of endocarditis and mortality were remarkably similar to those in other studies published since 1976.

Libman and Arbeit [22] reported a study of 37 patients with 39 episodes of SAB at the Boston Veterans Administration Medical Center between December 1980 and November 1981. In contrast to previous studies, the majority $(87 \%)$ of episodes were hospital acquired. Infected iv catheters produced $51 \%$ of the episodes, a much higher proportion than in any previous study. No patients with endocarditis were identified. This was probably related to the high frequency of iv-catheter-induced infections, which are known to have a good prognosis and a low risk of endocarditis $[17-19,31,36]$. On the other hand, metastatic complications occurred in $23 \%$ of the episodes, including those in four patients with catheter-associated SAB. However, in three of the four patients with catheter-associated $\mathrm{SAB}$ and metastatic infection, antibiotic therapy was delayed for 48-72 hours after blood cultures were obtained. This group with delayed therapy also had prolonged bacteremia (median duration, 4 days). Thus, it is not surprising that metastatic infection occurred in this group. The mortality rate directly due to SAB $(30 \%)$ was in agreement with our previous report [19] in a similar patient population.

Finkelstein and colleagues [23] reviewed the findings in 103 cases of SAB that occurred in a university hospital in Israel between January 1979 and December 1982 . No cases of endocarditis were recognized in those patients with $\mathrm{SAB}$ secondary to infected iv 
catheters. All but one patient with endocarditis had community-acquired SAB without a primary focus. The development of endocarditis in association with community-acquired SAB without a primary focus, a finding also reported by Mirimanoff and Glauser [18], supported the view of Nolan and Beaty [15] concerning the increased risk of endocarditis in this subgroup with $\mathrm{SAB}$ without the confounding factor of drug abuse. The disconcerting aspect of this study was the high mortality rate in patients with endocarditis $(64 \%)$ and the much higher overall rate of mortality $(43 \%)$ due to SAB than that reported in other recent studies (see table 9 [38]). The explanation for the excessive mortality in this study is not readily apparent.

Bryan and colleagues [24] retrospectively reviewed 390 consecutive episodes of SAB in four nonuniversity hospitals in Columbia, South Carolina, between January 1977 and December 1981. Endocarditis was related predominately to community-acquired SAB. Of note was that $26 \%$ of 35 patients with iv-catheterinduced $\mathrm{SAB}$ died. Although the findings in this study are in agreement with those in many previous studies in regard to the risk of endocarditis and mortality, one must be cautious in the interpretation of the data. Although not stated specifically in this study of SAB, Brenner and Bryan [39] and Bryan and colleagues [40] described in detail the four hospitals that were surveyed. These included a community hospital without a house staff and a Veterans Administration hospital. The combination of findings from various nonuniversity hospitals may not necessarily give a true picture of SAB. This is especially true when one combines data from a Veterans Administration hospital in which an elderly male population predominates with data from a community hospital with a more balanced population in terms of age.

Julander [25] retrospectively reviewed 273 patients with $\mathrm{SAB}$ with and without endocarditis who were admitted to a hospital for infectious diseases in Stockholm, Sweden. Unlike most other studies, which generally deal with a general hospital population, this study dealt with data from a specialized hospital. Thus, there may be a tendency for more complications in this setting. Also, $35 \%$ of the patients were drug addicts. Since this subgroup with a background of drug abuse is at increased risk for the development of endocarditis in the presence of $\mathrm{SAB}$ [15], it is not surprising that $31 \%$ of the patients had endocarditis.
Several trends are recognized in studies of $\mathrm{SAB}$ published since 1976 . First, SAB has become predominately a hospital-acquired infection. Second, infected iv catheters have become a prominent cause of SAB. However, the risk of endocarditis associated with iv-catheter-induced SAB was minimal except in one study [16]. Third, the rate of endocarditis was variable, with reported rates from $4 \%$ to $33 \%$. In several studies $[15,18,23]$ endocarditis occurred most commonly in the setting of community-acquired $\mathrm{SAB}$ without a primary focus. Fourth, there was no marked change in the mortality due to SAB compared with that in the previous period analyzed. Since most patients developing SAB have significant underlying diseases, and since potent antistaphylococcal therapy has been available for some time, there may be little or no further decrease in mortality.

\section{Discussion}

The findings in the current report can be summarized as follows. In the setting of a Veterans Administration medical center, SAB was predominately a hospital-acquired infection; the commonest source of SAB was an infected intravascular catheter; endocarditis and metastatic infection were infrequent, each occurring in $<5 \%$ of all cases; and mortality directly related to SAB was high $(32 \%)$. These findings are remarkably similar to those at the Boston VAMC reported by Libman and Arbeit [22].

One of the objectives of our study was to examine the risk of endocarditis in patients with communityacquired SAB without a definite focus of infection. Since only six of the 114 episodes were in this category, it was not possible to make any valid conclusions about the risk of endocarditis in this group from our data. However, on the basis of the findings of other studies $[15,18,23]$, one must be concerned about the possibility of an increased risk of endocarditis in this subgroup of patients with SAB. Until more prospective studies are reported in which the risk of endocarditis is carefully examined in a large number of patients with community-acquired $\mathrm{SAB}$, one must rely on the available information and strongly consider prolonged (4- to 6-week) parenteral antimicrobial therapy in this group of patients. Along these lines, Bayer et al. [41] have recently published in abstract form the results of a prospective survey of patients with $\mathrm{SAB}$ at the HarborviewUCLA Medical Center. Using multivariate logistic analysis, the authors reported that several variables - 
including community-acquired $\mathrm{SAB}$ and the absence of a primary focus - significantly predicted endocarditis. Details concerning the population studied were not given; therefore, the importance of these observations awaits full publication of their findings.

In the present study there were 23 episodes $(20 \%)$ of $\mathrm{SAB}$ in which the focus of infection was not identified. In other published reports of SAB, 10\%-40\% of all episodes of SAB had no identifiable focus (tables 7-9). Of the 23 episodes of SAB in the present study, $17(74 \%)$ were hospital-acquired infections. Despite close evaluation of these patients, no definite focus could be verified. It is possible that some episodes were related to infection of intravascular catheters that were undetected because of lack of signs of infection or failure to culture catheters. However, the source of SAB in this subgroup remains unclear.

It is possible that some patients in the present study who died had endocarditis or metastatic infection that was unrecognized. On the other hand, most of the patients who died had hospital-acquired SAB $(31$ of $36 ; 86 \%)$ and were promptly treated. We believe that this would tend to decrease the rate of complications. In addition, factors known to predispose to nosocomial endocarditis, e.g., rheumatic valvular disease and prosthetic valves [42], were not present in our population to any great extent. Nevertheless, because few autopsies were performed, the rate of endocarditis may be underestimated in the present study.

It is also possible that some patients who completed a planned course of therapy for SAB had endocarditis or metastatic infection that was unrecognized but successfully treated. However, this is unlikely in our opinion. The mean duration of therapy in our patients who recovered was 16.4 days. It has been found that patients with $S$. aureus endocarditis treated for $<4$ weeks have a high likelihood of relapse [15]. Thus, one would have expected some relapses on follow-up in those who initially recovered. However, no relapses occurred among 40 patients with a mean duration of follow-up of 34.8 weeks after completion of treatment.

We believe it is now clear that there is a low risk of endocarditis in patients with $\mathrm{SAB}$ related to an infected intravascular device if there is prompt removal of the infected device and antibiotic treatment. The findings in the present report as well as in several others $[17-21,31,36]$ have verified this.
Thus, for patients with intravascular device-related $\mathrm{SAB}$, we currently recommend 2 weeks of parenteral antimicrobial therapy along with removal of the device. However, it should be kept in mind that a small number of patients with catheter-associated $\mathrm{SAB}$ have been reported in whom complications other than endocarditis have developed during or after therapy $[18,22,29]$. It appears that such cases are the exception rather than the rule. In at least one study [22], delay in initiating therapy and prolonged bacteremia may have been factors that increased the risk of complications. Nevertheless, such complications have not always been clinically detectable early in the course. Therefore, Rahal et al. [43] have recently suggested a 4-week regimen (iv antibiotic therapy for 2 weeks followed by oral therapy for 2 weeks) as an alternative to short-course therapy ( 2 weeks of parenteral therapy) for patients with $\mathrm{SAB}$ due to a removable focus of infection or localized infection, e.g., skin. In our experience ([19], present report) the necessity to extend the duration of therapy beyond 14 days for catheter-associated $\mathrm{SAB}$ or $\mathrm{SAB}$ with a defined focus is infrequent. However, we agree with Rahal et al. [43] that if the clinical situation is such that host response is altered, e.g., the presence of malignancy, immunosuppression, delayed recognition of infection, or persistent bacteremia or fever, it would be prudent to prolong therapy beyond 2 weeks. In this situation the 4-week regimen suggested by Rahal et al. [43] is a reasonable approach.

It is of concern that infections of intravascular devices have become the single commonest cause of $\mathrm{SAB}$ at our institution as well as at other hospitals [21-23]. Theoretically, most of these infections are preventable if catheters are cared for properly [44]. There is evidence that a team dedicated to insertion and care of iv catheters can have an impact on the infection rate [45]. However, a major problem is recognition of catheter infection. In the case of infection of catheters in peripheral vessels, infection is often associated with phlebitis that is readily detectable clinically. Recognition of infection of central venous catheters is more problematic since evidence of inflammation at the insertion site is uncommon because of the depth of the subclavian vein below the skin [46]. This observation is confirmed in the present study in which no infected subclavian vein catheters had associated inflammation at the insertion site. This finding emphasizes the importance of considering an intravascular catheter as the source 
of sepsis or bacteremia even in the absence of local signs of infection.

Despite the lack of recognizable complications of $\mathrm{SAB}$ in our population, the mortality rate of $32 \%$ due to infection is disconcerting. There are several explanations for this finding. First, our hospital population is an elderly one for the most part; the mean age of patients with SAB was 64 years. It has been previously reported in several studies of SAB that patients in this older age group have a poorer prognosis than do younger individuals $[12,13,16$, $18,23,24]$. Second, some patients with MRSA bacteremia did not receive vancomycin initially, and this was associated with a higher mortality than that of patients treated with vancomycin, a finding that has also been previously described [47]. Third, almost all of our patients had one or more significant underlying diseases that may, to various degrees, have impacted on the response to infection.

Review of the literature dealing with $\mathrm{SAB}$ in the general hospital setting indicated variation in the reported rate of endocarditis, metastatic complications, and mortality. This variability points out the necessity for caution in interpreting the findings of a particular study as being representative of what one might find in all hospital populations. This variability is exemplified by two studies $[14,15]$ that revealed considerably different frequencies of community-acquired SAB, rates of endocarditis, and mortality (see table 8) yet were done in the same geographic area and during approximately the same period.

Variability in the rate of endocarditis may also be related to the criteria used to make this diagnosis. Review of the criteria used in each study revealed some differences, especially in the literature before 1960. In these earlier studies, however, the diagnosis of endocarditis was confirmed by autopsy in many instances. In almost all studies of SAB published since 1960 , the criteria for the diagnosis of endocarditis were clearly stated. On examination of the rates of endocarditis reported in the studies listed in tables $7-9$, one finds that all but four studies $[6,15$, $18,25]$ reported a rate of endocarditis of $\leqslant 20 \%$ and most were in the range of $5 \%-15 \%$. In three of the four studies in which the rate of endocarditis exceeded $20 \%$, the patient population consisted, in part, of those with an increased risk of endocarditis - either drug addicts $[15,25]$ or those with valvular heart disease [6]. The report of Mirimanoff and
Glauser [18] stands alone as the only study in which a rate of endocarditis exceeding $20 \%$ was found with no predisposing risk factors for endocarditis. If these four studies are excluded, the rate of endocarditis reported in association with SAB has actually been fairly constant for the past 45 years.

MRSA bacteremia has become endemic at the Buffalo VAMC since introduction of the index case in June 1982 (authors' unpublished data). This index case was unusual in that the patient was hospitalized for several weeks before development of MRSA bacteremia. Previous studies of MRSA have emphasized that the patient with the index case was usually transferred from another hospital or had a community-acquired infection [48, 49]. An exception was presented in the recent report by Rimland [50] in which the index case of MRSA infection was acquired or developed in the hospital. The persistence of MRSA as a cause of bacteremia at the Buffalo VAMC is characteristic of this pathogen [51].

The mortality rate of $32 \%$ in patients with MRSA bacteremia treated with vancomycin was similar to that found in a study by Craven et al. [52]. Other reports [49] have demonstrated a lower mortality rate, but the patients with MRSA bacteremia were younger than our patients and those of Craven et al. [52]. The mortality rate in our patients with MRSA bacteremia not treated with vancomycin was higher than in those receiving vancomycin, a phenomenon that has also been previously reported [47].

In summary, several conclusions can be drawn from analysis of studies of SAB. (I) Intravenouscatheter infection has become the commonest cause of SAB but is associated with a low risk of endocarditis; effective therapy includes prompt catheter removal and parenteral antibiotic therapy for 2 weeks. (2) Endocarditis is a frequent complication of SAB in the drug addict and in patients with valvular heart disease; such patients should be treated as if endocarditis is present regardless of signs and symptoms. (3) The risk of endocarditis appears to be high in patients with community-acquired $\mathrm{SAB}$ without a definable focus; in this situation it seems prudent to advise antibiotic treatment for 4-6 weeks. (4) In the absence of clinical risk factors for endocarditis, impaired host response, or infections known to require prolonged therapy (e.g., osteomyelitis), patients with $\mathrm{SAB}$ due to defined foci (other than intravascular catheters) can be effectively treated for 2-3 weeks with parenteral antibiotics. At 
present, we recommend parenteral therapy in the hospital setting, where patients can be monitored for complications.

\section{Addendum}

Subsequent to the acceptance of this manuscript for publication, the complete report by Bayer et al. of data previously available only in abstract form [41] was published [53]. Seventy-two patients with SAB at the Harbor-UCLA Medical Center were followed prospectively between 1980 and 1984 . An unknown number of patients with $\mathrm{SAB}$ during the study period were excluded. Thus, although the study was prospective, the population evaluated represented only a subset of all patients with SAB. This factor makes results difficult to interpret. For example, although the site of acquisition of SAB is not clearly stated, it appears that the majority of cases were community acquired. Patient-selection criteria and the presence of drug addicts in the patient population probably produced this predominance of community-acquired cases. As we have pointed out (table 9), in the studies published since 1976 from institutions in the United States, SAB has been predominately nosocomial in origin. Thus, it is not surprising that the results in terms of endocarditis compared favorably with those of Nolan and Beaty [15] in which the patient population with $\mathrm{SAB}$ was very similar. Nevertheless, the study of Bayer et al. provided evidence that two-dimensional echocardiography may be useful in identifying endocarditis in patients with community-acquired $\mathrm{SAB}$ with a primary focus. In concurrence with many previous studies and the present one, endocarditis was not associated with SAB secondary to an infected intravascular catheter.

\section{References}

1. Sheagren JN. Staphylococcus aureus: the persistent pathogen. N Engl J Med 1984;310:1368-73, 1437-42

2. McGowan JE Jr, Barnes MW, Finland M. Bacteremia at Boston City Hospital: occurrence and mortality during 12 selected years (1935-1972), with special reference to hospital-acquired cases. J Infect Dis 1975;132:316-35

3. Weinstein MP, Murphy JR, Reller LB, Lichtenstein KA. The clinical significance of positive blood cultures: a comprehensive analysis of 500 episodes of bacteremia and fungemia in adults. II. Clinical observations, with special reference to factors influencing prognosis. Rev Infect Dis 1983;5:54-70

4. Centers for Disease Control. Nosocomial infection surveil- lance, 1984. In: CDC surveillance summaries. MMWR 1986;35(1SS):17SS-29SS

5. Skinner D, Keefer CS. Significance of bacteremia caused by Staphylococcus aureus: a study of one hundred and twentytwo cases and a review of the literature concerned with experimental infection in animals. Arch Intern Med 1941;68:851-75

6. Wilson R, Hamburger M. Fifteen years' experience with staphylococcus septicemia in a large city hospital: analysis of fifty-five cases in the Cincinnati General Hospital 1940-1955. Am J Med 1957;22:437-57

7. Faber V, Jessen O, Rosendal K, Eriksen KR. Staphylococcal bacteraemia. Clinical and bacteriological observations in 201 cases. Br Med J 1960;2:1832-6

8. Shirger A, Martin WJ, Nichols DR. Micrococcal bacteremia without endocarditis: clinical data and therapeutic considerations in 109 cases. Ann Intern Med 1957;47:39-48

9. Waisbren BA, Abboud F. Bacteremia due to coagulase-positive Staphylococcus aureus. Ann Intern Med 1960;52:643-67

10. Smith IM, Vickers AB. Natural history of 338 treated and untreated patients with staphylococcal septicaemia (1936-1955). Lancet 1960;1:1318-22

11. Hassall JE, Rountree PM. Staphylococcal septicaemia. Lancet 1959;1:213-7

12. Cluff LE, Reynolds RC, Page DL, Breckenridge JL. Staphylococcal bacteremia and altered host resistance. Ann Intern Med 1968;69:859-73

13. Jessen O, Rosendal K, Bülow P, Faber V, Eriksen KR. Changing staphylococci and staphylococcal infections: a ten-year study of bacteria and cases of bacteremia. N Engl J Med 1969;281:627-35

14. Austin TW, Wallace JF. Staphylococcus aureus bacteremia: a critical review of its treatment and association with infective endocarditis. Infection 1973;1:214-7

15. Nolan CM, Beaty HN. Staphylococcus aureus bacteremia: current clinical patterns. Am J Med 1976;60:495-500

16. Shah M, Watanakunakorn C. Changing patterns of Staphylococcus aureus bacteremia. Am J Med Sci 1979; 278:115-21

17. Cooper G, Platt R. Staphylococcus aureus bacteremia in diabetic patients: endocarditis and mortality. Am J Med 1982;73:658-62

18. Mirimanoff RO, Glauser MP. Endocarditis during Staphylococcus aureus septicemia in a population of non-drug addicts. Arch Intern Med 1982;142:1311-3

19. Mylotte JM, Beam TR Jr, Allen JC. Staphylococcus aureus bacteremia: a prospective study. South Med J 1983;76: 1132-5

20. Hedstrom SA, Christensson B. Staphylococcus aureus septicaemia and endocarditis at the University Hospital in Lund 1976-1980. Scand J Infect Dis 1983;41(Suppl):38-46

21. Gransden WR, Eykyn SJ, Phillips I. Staphylococcus aureus bacteraemia: 400 episodes in St. Thomas's Hospital. Br Med J 1984;288:300-3

22. Libman H, Arbeit RD. Complications associated with Staphylococcus aureus bacteremia. Arch Intern Med 1984;144: 541-5

23. Finkelstein R, Sobel JD, Nagler A, Merzbach D. Staphylococcus aureus bacteremia and endocarditis: comparison of nosocomial and community-acquired infection. J Med 1984;15:193-211

24. Bryan CS, Kirkhart B, Brenner ER. Staphylococcal bacter- 
emia: current patterns in nonuniversity hospitals. South Med J 1984;77:693-6

25. Julander I. Unfavorable prognostic factors in Staphylococcus aureus septicemia and endocarditis. Scand J Infect Dis 1985;17:179-87

26. Bauer AW, Kirby WMM, Sherris JC, Turck M. Antibiotic susceptibility testing by a standardized single disk method. Am J Clin Pathol 1966;45:493-6

27. Thornsberry C, Caruthers JQ, Baker CN. Effect of temperature on the in vitro susceptibility of Staphylococcus aureus to penicillinase-resistant penicillins. Antimicrob Agents Chemother 1973;4:263-9

28. Maki DG, Weise CE, Sarafin HW. A semiquantitative culture method for identifying intravenous-catheter-related infection. N Engl J Med 1977;296:1305-9

29. Bernhardt LL, Antopol SC, Simberkoff MS, Rahal JJ Jr. Association of teichoic acid antibody with metastatic sequelae of catheter-associated Staphylococcus aureus bacteremia: a failure of the two-week antibiotic treatment. Am J Med 1979;66:355-7

30. Kirchhoff LV, Sheagren JN. Epidemiology and clinical significance of blood cultures positive for coagulase-negative staphylococcus. Infect Control 1985;6:479-86

31. Mylotte JM, McDermott C. Staphylococcus aureus bacteremia caused by infected intravenous catheters. Am J Infect Control 1987;15:1-6

32. Lerner PI, Weinstein L. Infective endocarditis in the antibiotic era. N Engl J Med 1966;274:199-206, 259-66, 388-93

33. Tuazon CU, Cardella TA, Sheagren JN. Staphylococcal endocarditis in drug users. Clinical and microbiologic aspects. Arch Intern Med 1975;135:1555-61

34. Chambers HF, Korzeniowski OM, Sande MA. Staphylococcus aureus endocarditis: clinical manifestations in addicts and nonaddicts. Medicine (Baltimore) 1983;62:170-7

35. Abrams B, Sklaver A, Hoffman T, Greenman R. Single or combination therapy of staphylococcal endocarditis in intravenous drug abusers. Ann Intern Med 1979;90:789-91

36. Iannini PB, Crossley K. Therapy of Staphylococcus aureus bacteremia associated with a removable focus of infection. Ann Intern Med 1976;84:558-60

37. Watanakunakorn C, Baird IM. Staphylococcus aureus bacteremia and endocarditis associated with a removable infected intravenous device. Am J Med 1977;63:253-6

38. Watanakunakorn C, Baird IM. Prognostic factors in Staphylococcus aureus endocarditis and results of therapy with a penicillin and gentamicin. Am J Med Sci 1977;273:133-9

39. Brenner ER, Bryan CS. Nosocomial bacteremia in perspective: a community-wide study. Infect Control 1981;2:219-26

40. Bryan CS, Reynolds KL, Brenner ER. Analysis of 1,186 episodes of gram-negative bacteremia in non-university hospitals: the effects of antimicrobial therapy. Rev Infect Dis 1983;5:629-38
41. Bayer AS, Lam K, Ginzton L, Chiu CY, Norman DC, Ward JI. Staphylococcus aureus bacteremia - clinical, serological, and echocardiographic findings in patients with and without endocarditis. Clin Res 1986;34:49A

42. Friedland G, von Reyn F, Levy B, Arbeit R, Dasse P, Crumpacker C. Nosocomial endocarditis. Infect Control 1984;5:284-8

43. Rahal JJ Jr, Chan Y-K, Johnson G. Relationship of staphylococcal tolerance, teichoic acid antibody, and serum bactericidal activity to therapeutic outcome in Staphylococcus aureus bacteremia. Am J Med 1986;81:43-52

44. Simmons BP, Hooton TM, Wong ES, Allen JR. Guidelines for prevention of intravascular infections. Infect Control 1982;3:61-7

45. Tomford JW, Hershey CO, McLaren CE, Proter DK, Cohen DL. Intravenous therapy team and peripheral venous catheter-associated complications: a prospective controlled study. Arch Intern Med 1984;144:1191-4

46. Collignon PJ, Soni N, Pearson IY, Woods WP, Munro R, Sorrell TC. Is semiquantitative culture of central vein catheter tips useful in the diagnosis of catheter-associated bacteremia? J Clin Microbiol 1986;24:532-5

47. Myers JP, Linnemann CC Jr. Bacteremia due to methicillinresistant Staphylococcus aureus. J Infect Dis 1982;145: $532-6$

48. Locksley RM, Cohen ML, Quinn TC, Tompkins LS, Coyle MB, Kirihara JM, Counts GW. Multiply antibiotic-resistant Staphylococcus aureus: introduction, transmission, and evolution of nosocomial infection. Ann Intern Med 1982;97:317-24

49. Saravolatz LD, Pohlod DJ, Arking LM. Community-acquired methicillin-resistant Staphylococcus aureus infections: a new source for nosocomial outbreaks. Ann Intern Med 1982;97:325-9

50. Rimland D. Nosocomial infections with methicillin and tobramycin resistant Staphylococcus aureus-implication of physiotherapy in hospital-wide dissemination. Am J Med Sci $1985 ; 290: 91-2$

51. Boyce JM, Landry M, Deetz TR, DuPont HL. Epidemiologic studies of an outbreak of nosocomial methicillin-resistant Staphylococcus aureus infections. Infect Control 1981;2: $110-6$

52. Craven DE, Kollisch NR, Hsieh CR, Connolly MG Jr McCabe WR. Vancomycin treatment of bacteremia caused by oxacillin-resistant Staphylococcus aureus: comparison with $\beta$-lactam antibiotic treatment of bacteremia caused by oxacillin-sensitive Staphylococcus aureus. J Infect Dis 1983;147:137-43

53. Bayer AS, Lam K, Ginzton L, Norman DC, Chiu CY, Ward JI. Staphylococcus aureus bacteremia: clinical, serologic and echocardiographic findings in patients with and without endocarditis. Arch Intern Med 1987;147:457-62 